\title{
Autologous Bone Marrow-Derived Stem Cells for Treating Diabetic Neuropathy in Metabolic Syndrome
}

\author{
Wei Liu, ${ }^{1}$ Fengchun Yu, ${ }^{1}$ Zhenghong Zhou, ${ }^{1}$ Yi-Chen Li, ${ }^{2,3}$ \\ Dongsheng Fan, ${ }^{4}$ and Kai Zhu ${ }^{5,6}$ \\ ${ }^{1}$ Department of Neurology, Beijing Haidian Hospital, Beijing 100080, China \\ ${ }^{2}$ Division of Engineering in Medicine, Brigham and Women's Hospital, Harvard Medical School, Cambridge, MA 02139, USA \\ ${ }^{3}$ Harvard-MIT Division of Health Sciences and Technology, Massachusetts Institute of Technology, \\ Cambridge, MA 02139, USA \\ ${ }^{4}$ Department of Neurology, Peking University Third Hospital, Beijing 100191, China \\ ${ }^{5}$ Department of Cardiac Surgery, Zhongshan Hospital, Fudan University, Shanghai 200032, China \\ ${ }^{6}$ Shanghai Institute of Cardiovascular Disease, Shanghai 200032, China
}

Correspondence should be addressed to Dongsheng Fan; dsfan2010@aliyun.com and Kai Zhu; zhu.kail@zs-hospital.sh.cn

Received 2 June 2017; Accepted 23 August 2017; Published 1 October 2017

Academic Editor: James L. Sherley

Copyright (C) 2017 Wei Liu et al. This is an open access article distributed under the Creative Commons Attribution License, which permits unrestricted use, distribution, and reproduction in any medium, provided the original work is properly cited.

\begin{abstract}
Diabetic neuropathy is one of the most common and serious complications of diabetes mellitus and metabolic syndrome. The current therapy strategies, including glucose control and pain management, are not effective for most patients. Growing evidence suggests that infiltration of inflammation factors and deficiency of local neurotrophic and angiogenic factors contribute significantly to the pathologies of diabetic neuropathy. Experimental and clinical studies have shown that bone marrow-derived stem cells (BMCs) therapy represents a novel and promising strategy for tissue repair through paracrine secretion of multiple cytokines, which has a potential to inhibit inflammation and promote angiogenesis and neurotrophy in diabetic neuropathy. In this review, we discuss the clinical practice in diabetic neuropathy and the therapeutic effect of BMC. We subsequently illustrate the functional impairment of autologous BMCs due to the interrupted bone marrow niche in diabetic neuropathy. We anticipate that the functional restoration of BMCs could improve their therapeutic effect and enable their wide applications in diabetic neuropathy.
\end{abstract}

\section{Introduction}

Diabetic neuropathy is one of the most frequent complications in diabetes mellitus and metabolic syndromes, which cause high rate of disability and mortality $[1,2]$. Diabetic neuropathy can affect a wide spectrum of peripheral nerves, including pain fibres and motoneuron and autonomic nervous system [3]. However, its pathophysiology is not yet fully elucidated. The treatment of hyperglycemia is a recommended strategy to reduce the incidence of diabetes neuropathy [4]. However, it showed a marginal effect on preventing diabetic neuropathy in type 2 diabetes mellitus, indicating that other factors might be also involved in the nerve injury in these patients [4]. The other conventional therapeutics, such as aldose reductase inhibitor or $\alpha$-lipoic acid, also showed limited therapy response in the progressive stage of diabetes neuropathy [5]. Recently, it is speculated that diabetic neuropathy is secondary to the deficiency of local growth factors, besides which, a number of inflammatory mediators, such as interleukins and chemokines, are involved in the progression of diabetic neuropathy [6]. There is a growing interest in the scientific community for cellular therapies, such as utilizing of BMCs, for the treatment of diabetes and its complications [7]. In the past decade, BMCs have been applied as a promising therapy for diabetic neuropathy because of their multipotency and their comprehensive paracrine secretion of anti-inflammatory cytokines, proangiogenic factors, and neurotrophic factors [8]. However, diabetes neuropathy may jeopardize the physiological function of autologous BMCs, yielding their modest therapy 


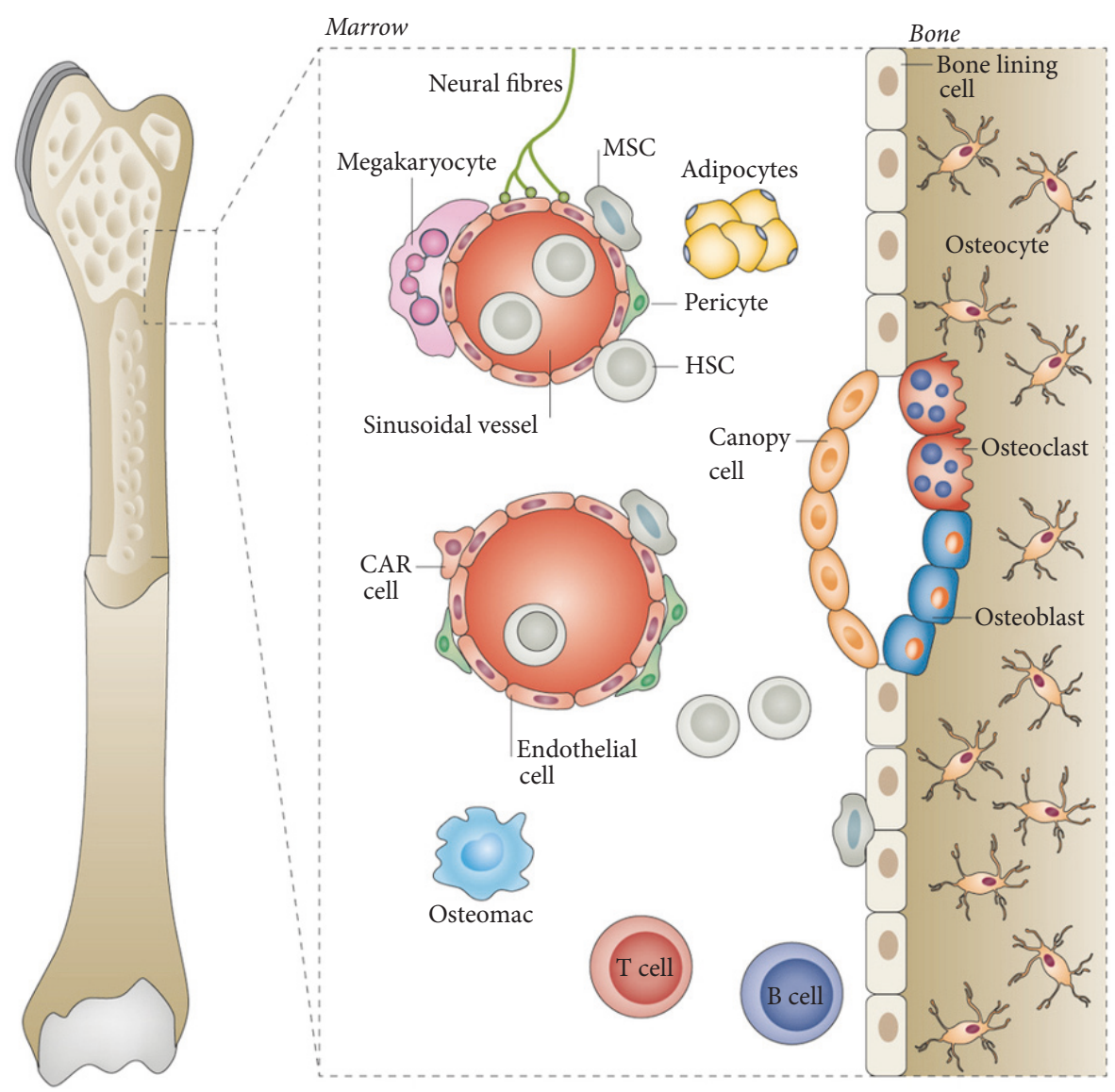

Figure 1: Schematic of bone marrow niche. Bone marrow niche is anatomical compartments constituted by cellular and extracellular components that mediate stem cell maintenance and expansion. Adapted by permission from [45] copyright Nature Publishing Group 2016.

efficacy [9]. Here, we review the clinical profile of diabetic neuropathy and BMC therapy. Finally, some solutions to restore BMC function in diabetes mellitus are addressed.

\section{Current Clinical Management of Diabetic Neuropathy}

Diabetic neuropathy has been defined as the presence of symptoms and/or signs of peripheral nerve dysfunction in people with diabetes after the exclusion of other causes, which is the most common complication of diabetes, affecting $30-50 \%$ of individuals with diabetes mellitus. Patients with diabetic neuropathy have poor quality of life and reduced work productivity, along with high healthcare costs. Cardiac autonomic neuropathy has been hailed as the "Prophet of Doom" and accounts for $20-34 \%$ of all patients with DM [10]. It is conducive to increased risk of myocardial infarction, malignant arrhythmia, and sudden death.

Diabetic neuropathy has been known for over two decades. The pathological mechanisms have been shown to be complicated (Figure 1). Diabetes mellitus often cause several prolonged metabolic disorders, including hyperglycemia, hyperinsulinemia, growth factor abnormalities, and dyslipidemia. These disorders could bring a complicated alternation, such as alteration of polyol metabolism and excessive oxidative stress, which could be triggered for the potential antigenic leakage and damage in nerve fibres. As a result, organic and structural nerve damage could occur. However, the regeneration capacity in nerve is limited, causing the irreversible neuropathy in patients with diabetes mellitus. Several therapeutics, such as aldose reductase inhibitor and $\alpha$-lipoic acid, have been developed based on these mechanisms. For instance, data from clinical trials showed that aldose reductase could improve the conduction velocity of motor and sensory nerves [11]. Nevertheless, the other involved mechanisms, such as nonenzymatic glucosylation, could be also targeted to develop novel therapeutics in future.

The high quality clinical evidence from 17 randomized studies ( 7 in type 1 diabetes mellitus, 8 in type 2 diabetes mellitus, and 2 in both types) showed that strict glucose control significantly prevented the development of diabetic neuropathy in patients with type 1 diabetes mellitus [12]. In contrast, the strict glucose control could reduce the incidence of neuropathy in type 2 diabetes mellitus, but without statistical significance $(P=0.06)$. However, the benefit from the aggressive glucose control has to be balanced against the increased risk of low blood glucose level, which could be dangerous and may lead to brain injury.

Diabetes mellitus also could lead to the autoimmune abnormality, which is a major cause of inflammation in the 
nerves and subsequent nerve damage. In one recent study, researchers found that blood serum autoimmune antibodies were significantly present in the patients with peripheral diabetic neuropathy [13]. Microvascular insufficiency in diabetes mellitus may also contribute to the pathogenetic mechanism in nerve neuropathy. The adequate blood supply from microvessels is crucial to maintain the normal structure and function in nerves. The strict blood glucose control is applied in patients with diabetes mellitus [4]. Poor glycemic control is clearly associated with the development of diabetic neuropathy. In addition, it has been found that diabetes could reduce neurotrophic factors in peripheral nerves by reducing the anterograde and retrograde axonal transport, thus making significant influence on neural morphology and conductivity. A meta-analysis including ten studies showed aldose reductase inhibitor improved cardiac autonomic function, with an acceptable safety profile for all agents except tolrestat [14].

\section{Characteristics of BMCs}

BMCs constitute a heterogeneous cell population, mainly including hematopoietic stem cells (HSCs), mesenchymal stem cells (MSCs), and endothelial progenitor cells (EPCs) [15]. In response to tissue injury or exogenously by cytokine stimulation, the endogenous BMCs can be mobilized to conduct their repair function [16]. The isolated BMCs can be cultured and expanded for implantation therapy [17-23]. Autologous BMCs are being explored in early clinical trials as therapy for various health disorders owing to no requirement for systemic immunosuppression or donor matching [24]. Collectively, the mobilized, intravascularly or locally delivered BMSCs can home into the damaged tissues or organs.

HSCs give rise to both the myeloid and lymphoid lineages of blood cells $[25,26]$. However, HSCs are also a large pool of cell population. The identification of HSCs is usually dependent on the specific cell surface markers, such as CD34 and CD133. For instance, CD34+ HSCs have been widely used in clinical trials to reconstitute the deficient hematopoietic system after radiation or chemotherapy [25]. Besides that, it has also been found that CD34+ HSCs could promote therapeutic angiogenesis in tissue ischemia models [27]. One of the underlying mechanisms is their paracrine secretion of angiogenic growth factors that induce microvasculature [28].

Mesenchymal stem cells (MSCs) can be isolated from bone marrow as well as in several other adult tissues [29]. They are highly proliferative and have capacity to differentiate into various cell types, thus making MSCs a promising candidate for a wide range of medicine applications. In recent studies, it has been found that implantation of allogeneic MSCs was protected from immune detection through "hit and run" (immune evasive) instead of hypoimmunogenic or immune privileged mechanisms [30]. In addition, the isolated MSCs could also be transfected with various therapeutic genes to exert improved functions after implantation [31].

EPCs are a subset of myeloid/monocyte cells whose surface can express endothelial markers, such as kinase insert domain receptor (KDR), CD31, CD133, or von Willebrand factor (vWf) [32]. Studies suggest that EPCs have favorable survival and a better response toward angiogenic growth factors compared with mature endothelial cells. The circulating EPCs concentration has been used for biomarkers for some disease detection and staging [33]. In addition, it has been demonstrated that EPCs have the benefit of proangiogenesis in ischemic tissues mainly via paracrine secretion rather than differentiation into vascular cells [34].

\section{BMC Therapy for Diabetic Neuropathy}

It has been found that growth factor therapy is an attractive option for diabetic neuropathy [35]. The administration of growth factors can promote angiogenesis and neural regeneration. Some growth factors, known as angioneurins, have both angiogenic and neurotrophic properties. In one study, researchers injected vascular endothelial growth factor (VEGF) encoding plasmids into diabetic animal models. The therapeutic showed enhancement in angiogenesis of vasa nervorum, increase in nerve fibre density, and normalization in nerve conductivity velocity [35]. A randomized, doubleblinded trial in humans showed that VEGF could evoke statistically significant symptomatic improvement of diabetic neuropathy [36]. Other growth factors, such as insulinlike growth factor (IGF), nerve growth factor (NGF), and ciliary neurotrophic factor, were also investigated in experimental animals with diabetic neuropathy [37]. They were found to ameliorate development of neuropathy and improve nerve function. Therefore, a therapeutic strategy to promote angiogenesis and neuron regeneration may be promising in treatment of diabetic neuropathy.

In this context, BMCs, which can produce multiple angiogenic and neurotrophic growth factors and potentially supplement specific type of cells required for vascular or neuron regeneration, have advantages over growth factor therapy [38]. Currently, preclinical studies showed their potential therapeutic effects in diabetic neuropathy. One of the advantages to use BMCs is the possibility of autograft; that is, they can be harvested from a patient and autologously administered back. In rat models with type 1 diabetes, bone marrow-derived MSCs were found to ameliorate hypoalgesia and normalize nerve conduction velocities [39]. Although MSCs have been proved to support de novo regeneration of neuronal cells, paracrine properties seem to be more prevalent. Besides neurotrophic and angiogenic factors such as NGF, VEGF, and IGF-1, MSCs can also produce antiinflammatory cytokines that moderate leukocyte recruitment in injured nerve [40]. This multiple-aspected action on inflammation, nerves, and vessels is also exerted by EPCs. In a type 1 diabetes mouse model, EPC administration increased the level of VEGF, basic fibroblast growth factor, and glioma-associated oncogene family zinc finger 1 protein, thus booming the proliferation of Schwann and endothelial cells [41]. Besides that, the endogenous cell mobilization is an alternative to cell transplantation therapy. In a rodent model with diabetic neuropathy, intraperitoneal administration of granulocyte colony-stimulating factor (G-CSF) recruited stem cells from bone marrow to improve nerve function [42]. However, no proof of fusion with local cells or neuron cyte differentiation was observed, thus indicating 
that a paracrine action was conducted by the recruited stem cells.

\section{Functional Impairment of BMC in Diabetic Neuropathy}

The reservoir of BMCs, namely, bone marrow niches, are anatomical compartments constituted by cellular and extracellular components. They are crucial for the stem cell maintenance and expansion (Figure 1) [43-45]. As functional entity with a high plasticity capacity, bone marrow niche is able to response rapidly for the signals from the body. Under aging and disease conditions, bone marrow niches could undergo extensive remodeling, thus altering the properties of resident BMCs. Interruption in bone marrow niche homeostasis may lead to abnormalities of stem cell renewal, lineage specification, and mobilization.

Sympathetic and nociceptive fibres stimulate the release of BMCs into bloodstream [46]. Recent report demonstrated that the occurrence of diabetic autonomic neuropathy might result in the defective mobilization of BMSCs [47]. In a clinical trial (NCT01102699), patients with diabetes mellitus showed impaired mobilization ability of HSCs and EPCs while being in response to G-CSF [48]. In another study, Albiero et al. demonstrated that both experimental murine models of type 1 and type 2 diabetes develop bone marrow autonomic neuropathy with an impaired mobilization of BMCs by upregulation of src homology and collagen homology domain and downregulation of Sirtuin 1 (Sirtl) [49]. Mobilization of vascular stem cells plays an important role in repairing tissue damage by promoting angiogenesis and reperfusion. Therefore, impaired mobilization of cells is expected to promote cardiovascular disease related to cardiac autonomic neuropathy. Collectively, these reports indicate that diabetic neuropathy may adversely affect stem cell-based therapies in patients with diabetic neuropathy.

\section{Functional Restoration of BMCs}

To improve BMC mobilization in diabetes, some types of cytokines could be alternative to G-CSF. In a previous study, researchers found that G-CSF failed to mobilize bone marrow stem cells in diabetic patients. However, when G-CSF was administrated in conjunction with plerixafor, an antagonist of the SDF-1/CXCR4 axis, the mobilization ability of stem cells was observed to be restored $[48,50]$.

For the ex vivo isolated BMSCs, genetic engineering could be an efficient strategy to rescue functionality of stem cells. It has been well known that cell development and function could be regulated through the modulation of miRNA level, thus enabling miRNA as the therapeutic target in BMCs $[51,52]$. For instance, downregulation of microRNA-155 in hematopoietic stem cells was found to maintain the pool of original stem cells in diabetic bone marrow [53]. In addition, downregulation of microRNA-15a and microRNA-16 could ameliorate angiogenic potential of diabetic EPCs [54].

Much evidence showed that function of BMCs from diabetes could be restored through administration of some gaseous signal molecule, such as hydrogen sulfide. In one study from Liu et al., hydrogen sulfide was found to restore the angiogenic functions of EPCs by upregulating expression of Ang-1 [55]. In a recent study, Cheng et al. demonstrated that decreased cystathionine $\gamma$-lyase-mediated hydrogen sulfide bioavailability is the potential reason of impairment of BMC function in diabetes [56]. They applied the hydrogen sulfide donor treatment or overexpression of CSE gene in diabetic BMCs and found improved repair capacity in diabetic critical limb ischemia.

Other strategies include adding of the culture media with a supplement of cytokines $[57,58]$. For instance, Kim et al. reported that Krüppel-like factor 2 (KLF2), a critical angiogenic factor, was dramatically decreased in diabetic bone marrow-derived MSCs [59]. The addition of oxytocin into the culture medium could significantly restore function of MSCs through upregulation of KLF-2 expression.

\section{Perspective and Conclusions}

As evidence grows on the safety of autologous BMC therapy in a number of clinical trials, the potential to translate this therapeutic strategy into a pilot clinical trial for diabetic neuropathy is very promising. However, several steps still need to be completed before that can be a reality. Firstly, our current knowledge of restoring the proper bone marrow niche microenvironment in patients with diabetes requires more investigation. The modulation of bone marrow niche is vital to obtain the healthy candidate cells. In addition, the isolated BMCs need to be functionally modified through genetic engineering and other strategies. In conclusion, BMC therapy may become a potential therapeutic option to treat diabetic neuropathy.

\section{Conflicts of Interest}

The authors declare that there are no conflicts of interest regarding the publication of this paper.

\section{Authors' Contributions}

Wei Liu and Fengchun Yu contributed equally to this work.

\section{Acknowledgments}

The authors acknowledge Dr. Qiu Zhong for the revision of this paper. Dr. Kai Zhu acknowledge funding from the National Science Foundation of China (Grant no. 81301312) and "Chen Guang" Project supported by Shanghai Municipal Education Commission and Shanghai Education Development Foundation (Grant no. 14CG06).

\section{References}

[1] E. L. Feldman, M. J. Stevens, P. K. Thomas, M. B. Brown, N. Canal, and D. A. Greene, "A practical two-step quantitative clinical and electrophysiological assessment for the diagnosis 
and staging of diabetic neuropathy," Diabetes Care, vol. 17, no. 11, pp. 1281-1289, 1994.

[2] G. Said, "Diabetic neuropathy-a review," Nature Clinical Practice Neurology, vol. 3, no. 6, pp. 331-340, 2007.

[3] P. J. Dyck, K. M. Kratz, J. L. Karnes et al., "The prevalence by staged severity of various types of diabetic neuropathy, retinopathy, and nephropathy in a population-based cohort: the Rochester Diabetic Neuropathy Study," Neurology, vol. 43, no. 4, pp. 817-824, 1993.

[4] B. C. Callaghan, H. T. Cheng, C. L. Stables, A. L. Smith, and E. L. Feldman, "Diabetic neuropathy: clinical manifestations and current treatments," The Lancet Neurology, vol. 11, no. 6, pp. 521534, 2012.

[5] D. Ziegler, M. Hanefeld, K. J. Ruhnau et al., "Treatment of symptomatic diabetic peripheral neuropathy with the antioxidant $\alpha$-lipoic acid - A 3-week multicentre randomized controlled trial (ALADIN Study)," Diabetologia, vol. 38, no. 12, pp. 1425-1433, 1995.

[6] R. Pop-Busui, L. Ang, C. Holmes, K. Gallagher, and E. L. Feldman, "Inflammation as a Therapeutic Target for Diabetic Neuropathies," Current Diabetes Reports, vol. 16, no. 3, article no. 29, pp. 1-10, 2016.

[7] M. A. Hussain and N. D. Theise, "Stem-cell therapy for diabetes mellitus," Lancet, vol. 364, no. 9429, pp. 203-209, 2004.

[8] H. Kim, J.-S. Park, J. C. Yong et al., "Bone marrow mononuclear cells have neurovascular tropism and improve diabetic neuropathy," Stem Cells, vol. 27, no. 7, pp. 1686-1696, 2009.

[9] L. Shin and D. A. Peterson, "Impaired therapeutic capacity of autologous stem cells in a model of type 2 diabetes," Stem Cells Translational Medicine, vol. 1, no. 2, pp. 125-135, 2012.

[10] D. Ziegler, F. A. Gries, M. Spüler, and F. Lessmann, "The epidemiology of diabetic neuropathy," Journal of Diabetes and Its Complications, vol. 6, no. 1, pp. 49-57, 1992.

[11] P. Boucek, "Advanced diabetic neuropathy: a point of no return," Review of Diabetic Studies, vol. 3, no. 3, pp. 143-150, 2006.

[12] B. C. Callaghan, A. A. Little, E. L. Feldman, and R. A. C. Hughes, "Enhanced glucose control for preventing and treating diabetic neuropathy," Cochrane Database of Systematic Reviews, vol. 13, no. 6, 2012.

[13] N. M. Janahi, D. Santos, C. Blyth, M. Bakhiet, and M. Ellis, "Diabetic peripheral neuropathy, is it an autoimmune disease?" Immunology Letters, vol. 168, no. 1, pp. 73-79, 2015.

[14] S. Javed, U. Alam, and R. A. Malik, "Treating diabetic neuropathy: present strategies and emerging solutions," Review of Diabetic Studies, vol. 12, no. 1-2, pp. 63-83, 2015.

[15] M. Ushio-Fukai and J. Rehman, "Redox and metabolic regulation of stem/progenitor cells and their niche," Antioxidants and Redox Signaling, vol. 21, no. 11, pp. 1587-1590, 2014.

[16] Y. Chen, L.-X. Xiang, J.-Z. Shao et al., "Recruitment of endogenous bone marrow mesenchymal stem cells towards injured liver," Journal of Cellular and Molecular Medicine, vol. 14, no. 6 B, pp. 1494-1508, 2010.

[17] K. Zhu, C. Guo, H. Lai, W. Yang, and C. Wang, "Novel hyperbranched polyamidoamine nanoparticle based gene delivery: Transfection, cytotoxicity and in vitro evaluation," International Journal of Pharmaceutics, vol. 423, no. 2, pp. 378-383, 2012.

[18] K. Zhu, H. Lai, C. Guo, D. Xu, and C. Wang, "Novel vascular endothelial growth factor gene delivery system-manipulated mesenchymal stem cells repair infarcted myocardium," Experimental Biology and Medicine, vol. 237, no. 6, pp. 678-687, 2012.
[19] Y. Xia, K. Zhu, H. Lai et al., "Enhanced infarct myocardium repair mediated by thermosensitive copolymer hydrogel-based stem cell transplantation," Experimental Biology and Medicine, vol. 240, no. 5, pp. 593-600, 2015.

[20] K. Zhu, M. Wu, H. Lai et al., "Nanoparticle-enhanced generation of gene-transfected mesenchymal stem cells for in vivo cardiac repair," Biomaterials, vol. 74, pp. 188-199, 2016.

[21] K. Zhu, C. Guo, J. Li et al., "Nanovector-based prolyl hydroxylase domain 2 silencing system enhances the efficiency of stem cell transplantation for infarcted myocardium repair," International Journal of Nanomedicine, vol. 9, no. 1, pp. 52035215, 2014.

[22] J. Li, K. Zhu, Y. Wang et al., "Combination of IGF-1 gene manipulation and 5-AZA treatment promotes differentiation of mesenchymal stem cells into cardiomyocyte-like cells," Molecular Medicine Reports, vol. 11, no. 2, pp. 815-820, 2015.

[23] J. Li, K. Zhu, S. Yang et al., "Fibrin patch-based insulin-like growth factor-1 gene-modified stem cell transplantation repairs ischemic myocardium," Experimental Biology and Medicine, vol. 240, no. 5, pp. 585-592, 2015.

[24] K. Zhu, J. Li, Y. Wang et al., "Intramyocardial Autologous Bone Marrow-derived Stem Cells Injection for Ischemic Heart Disease Ineligible for Revascularization: A Systematic Review and Meta-analysis," Archives of Medical Research, vol. 46, no. 4, pp. 286-295, 2015.

[25] M. Kondo, A. J. Wagers, M. G. Manz et al., "Biology of hematopoietic stem cells and progenitors: implications for clinical application," Annual Review of Immunology, vol. 21, pp. 759-806, 2003.

[26] E. L. Herzog, L. Chai, and D. S. Krause, "Plasticity of marrowderived stem cells," Blood, vol. 102, no. 10, pp. 3483-3493, 2003.

[27] N. Takakura, T. Watanabe, S. Suenobu et al., "A role for hematopoietic stem cells in promoting angiogenesis," Cell, vol. 102, no. 2, pp. 199-209, 2000.

[28] H. Kim, H.-J. Cho, S.-W. Kim et al., "CD31+ cells represent highly angiogenic and vasculogenic cells in bone marrow: Novel role of nonendothelial CD31+ cells in neovascularization and their therapeutic effects on ischemic vascular disease," Circulation Research, vol. 107, no. 5, pp. 602-614, 2010.

[29] M. Ohishi and E. Schipani, "Bone marrow mesenchymal stem cells," Journal of Cellular Biochemistry, vol. 109, no. 2, pp. 277282, 2010.

[30] J. A. Ankrum, J. F. Ong, and J. M. Karp, "Mesenchymal stem cells: immune evasive, not immune privileged," Nature Biotechnology, vol. 32, no. 3, pp. 252-260, 2014.

[31] K. Zhu, J. Li, Y. Wang, H. Lai, and C. Wang, "Nanoparticlesassisted stem cell therapy for ischemic heart disease," Stem Cells International, vol. 2016, Article ID 1384658, 2016.

[32] C. Urbich and S. Dimmeler, "Endothelial progenitor cells: characterization and role in vascular biology," Circulation Research, vol. 95, no. 4, pp. 343-353, 2004.

[33] M. A. Alaiti, M. Ishikawa, and M. A. Costa, "Bone marrow and circulating stem/progenitor cells for regenerative cardiovascular therapy," Translational Research, vol. 156, no. 3, pp. 112-129, 2010.

[34] C. Kalka, H. Masuda, T. Takahashi et al., "Transplantation of ex vivo expanded endothelial progenitor cells for therapeutic neovascularization," Proceedings of the National Academy of Sciences of the United States of America, vol. 97, no. 7, pp. 3422$3427,2000$. 
[35] P. Schratzberger, D. H. Walter, K. Rittig et al., "Reversal of experimental diabetic neuropathy by VEGF gene transfer," The Journal of Clinical Investigation, vol. 107, no. 9, pp. 1083-1092, 2001.

[36] A. H. Ropper, K. C. Gorson, C. L. Gooch et al., "Vascular Endothelial Growth Factor Gene Transfer for Diabetic Polyneuropathy: A Randomized, Double-Blinded Trial," Annals of Neurology, vol. 65, no. 4, pp. 386-393, 2009.

[37] M. Ii, H. Nishimura, K. F. Kusano et al., "Neuronal nitric oxide synthase mediates statin-induced restoration of vasa nervorum and reversal of diabetic neuropathy," Circulation, vol. 112, no. 1, pp. 93-102, 2005.

[38] J. W. Han, M. Y. Sin, and Y.-S. Yoon, "Cell therapy for diabetic neuropathy using adult stem or progenitor cells," Diabetes and Metabolism Journal, vol. 37, no. 2, pp. 91-105, 2013.

[39] T. Shibata, K. Naruse, H. Kamiya et al., "Transplantation of bone marrow-derived mesenchymal stem cells improves diabetic polyneuropathy in rats," Diabetes, vol. 57, no. 11, pp. 3099-3107, 2008.

[40] D. J. Prockop and J. Y. Oh, "Mesenchymal stem/stromal cells (MSCs): role as guardians of inflammation," Molecular Therapy, vol. 20, no. 1, pp. 14-20, 2012.

[41] J.-O. Jeong, M.-O. Kim, H. Kim et al., "Dual angiogenic and neurotrophic effects of bone marrow-derived endothelial progenitor cells on diabetic neuropathy," Circulation, vol. 119, no. 5, pp. 699-708, 2009.

[42] P. E. Westerweel, M. Teraa, S. Rafii et al., "Impaired Endothelial Progenitor Cell Mobilization and Dysfunctional Bone Marrow Stroma in Diabetes Mellitus," PLoS ONE, vol. 8, no. 3, Article ID e60357, 2013.

[43] S. J. Morrison and D. T. Scadden, "The bone marrow niche for haematopoietic stem cells," Nature, vol. 505, no. 7483, pp. 327334, 2014.

[44] R. N. Kaplan, B. Psaila, and D. Lyden, "Bone marrow cells in the 'pre-metastatic niche': Within bone and beyond," Cancer and Metastasis Reviews, vol. 25, no. 4, pp. 521-529, 2006.

[45] M. R. Reagan and C. J. Rosen, "Navigating the bone marrow niche: Translational insights and cancer-driven dysfunction," Nature Reviews Rheumatology, vol. 12, no. 3, pp. 154-168, 2016.

[46] J. Isern and S. Méndez-Ferrer, "Stem cell interactions in a bone marrow niche," Current Osteoporosis Reports, vol. 9, no. 4, pp. 210-218, 2011.

[47] F. Ferraro, S. Lymperi, S. Méndez-Ferrer et al., "Diabetes impairs hematopoietic stem cell mobilization by altering niche function," Science Translational Medicine, vol. 3, no. 104, 2011.

[48] G. P. Fadini, M. Albiero, S. V. de Kreutzenberg et al., "Diabetes impairs stem cell and proangiogenic cell mobilization in humans," Diabetes Care, vol. 36, no. 4, pp. 943-949, 2013.

[49] M. Albiero, N. Poncina, M. Tjwa et al., "Diabetes causes bone marrow autonomic neuropathy and impairs stem cell mobilization via dysregulated p66Shc and Sirt1," Diabetes, vol. 63, no. 4, pp. 1353-1365, 2014.

[50] G. P. Fadini, M. Fiala, R. Cappellari et al., "Diabetes limits stem cell mobilization following G-CSF but not plerixafor," Diabetes, vol. 64, no. 8, pp. 2969-2977, 2015.

[51] C. Chen, L. Li, H. F. Lodish, and D. P. Bartel, "MicroRNAs modulate hematopoietic lineage differentiation," Science, vol. 303, no. 5654, pp. 83-86, 2004.

[52] K. Zhu, D. Liu, H. Lai, J. Li, and C. Wang, "Developing miRNA therapeutics for cardiac repair in ischemic heart disease," Journal of Thoracic Disease, vol. 8, no. 9, pp. E918-E927, 2016.
[53] G. Spinetti, D. Cordella, O. Fortunato et al., "Global remodeling of the vascular stem cell niche in bone marrow of diabetic patients: implication of the microRNA-155/FOXO3a signaling pathway," Circulation Research, vol. 112, no. 3, pp. 510-522, 2013.

[54] N. M. Kane, A. J. Thrasher, G. D. Angelini, and C. Emanueli, "Concise review: MicroRNAs as modulators of stem cells and angiogenesis," Stem Cells, vol. 32, no. 5, pp. 1059-1066, 2014.

[55] F. Liu, D.-D. Chen, X. Sun et al., "Hydrogen sulfide improves wound healing via restoration of endothelial progenitor cell functions and activation of angiopoietin-1 in type 2 diabetes," Diabetes, vol. 63, no. 5, pp. 1763-1778, 2014.

[56] Z. Cheng, V. N. S. Garikipati, E. Nickoloff et al., "Restoration of Hydrogen Sulfide Production in Diabetic Mice Improves Reparative Function of Bone Marrow Cells," Circulation, vol. 134, no. 19, pp. 1467-1483, 2016.

[57] C. L. Smith, K. L. Chaichana, Y. M. Lee et al., "Pre-exposure of human adipose mesenchymal stem cells to soluble factors enhances their homing to brain cancer," Stem Cells Translational Medicine, vol. 4, no. 3, pp. 239-251, 2015.

[58] J. A. Zimmermann and T. C. Mcdevitt, "Pre-conditioning mesenchymal stromal cell spheroids for immunomodulatory paracrine factor secretion," Cytotherapy, vol. 16, no. 3, pp. 331345, 2014.

[59] Y. S. Kim, J. S. Kwon, M. H. Hong et al., "Restoration of angiogenic capacity of diabetes-insulted mesenchymal stem cells by oxytocin," BMC Cell Biology, vol. 14, no. 1, article 38, 2013. 


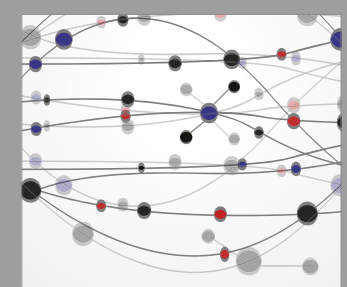

The Scientific World Journal
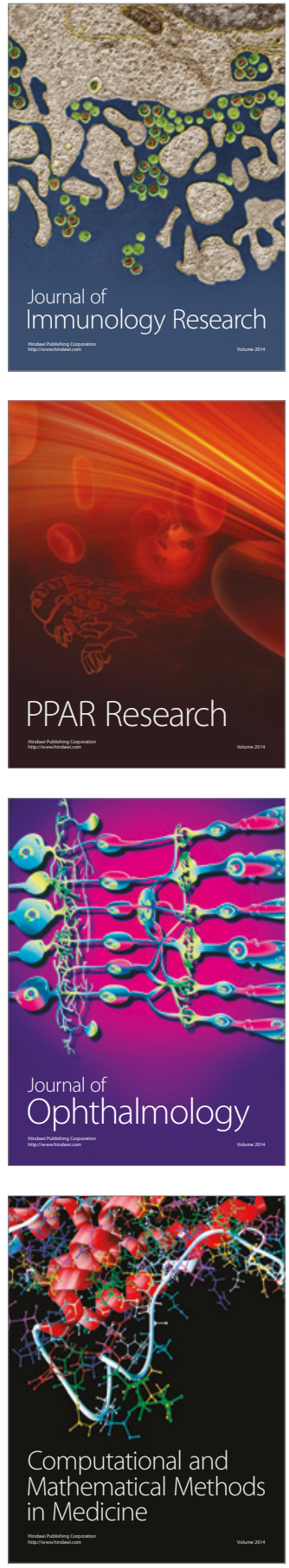

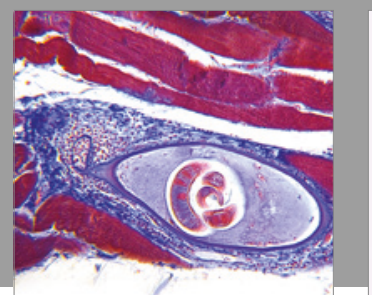

Gastroenterology Research and Practice
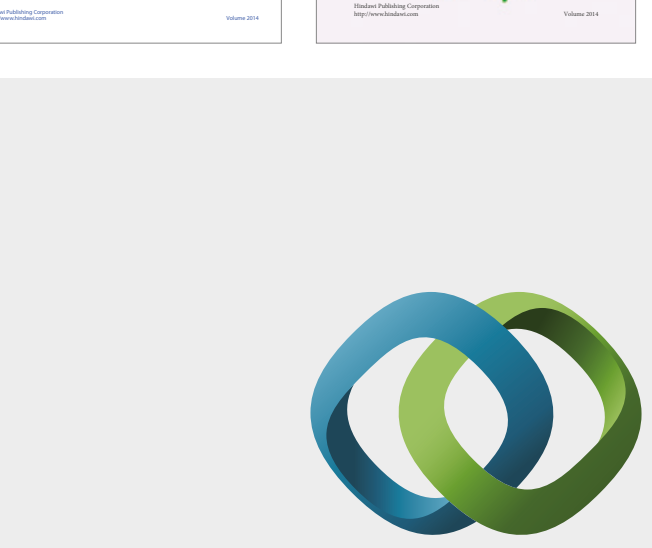

\section{Hindawi}

Submit your manuscripts at

https://www.hindawi.com
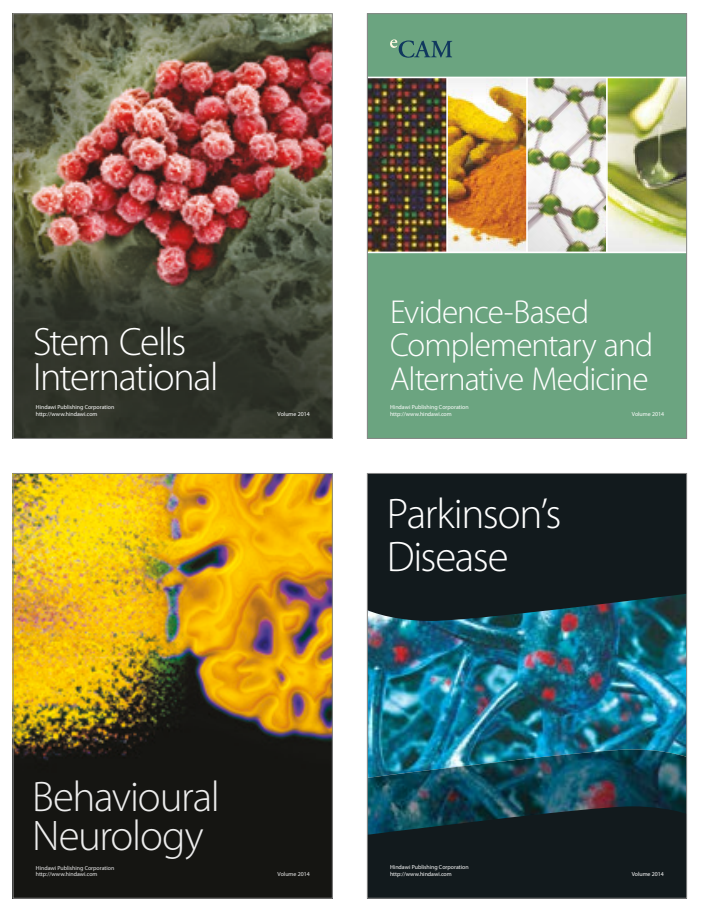
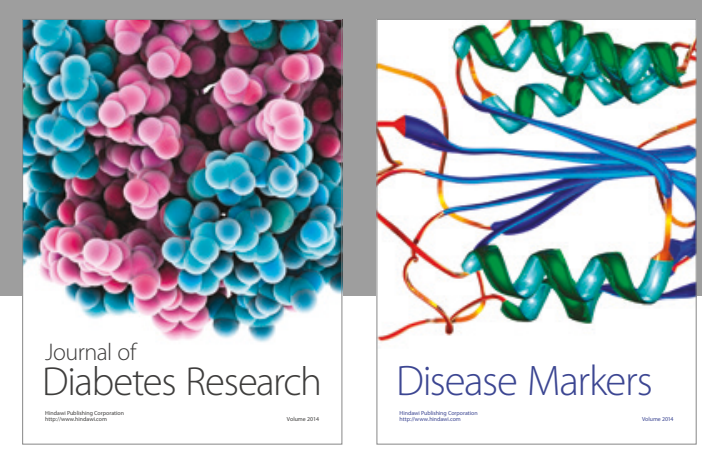

Disease Markers
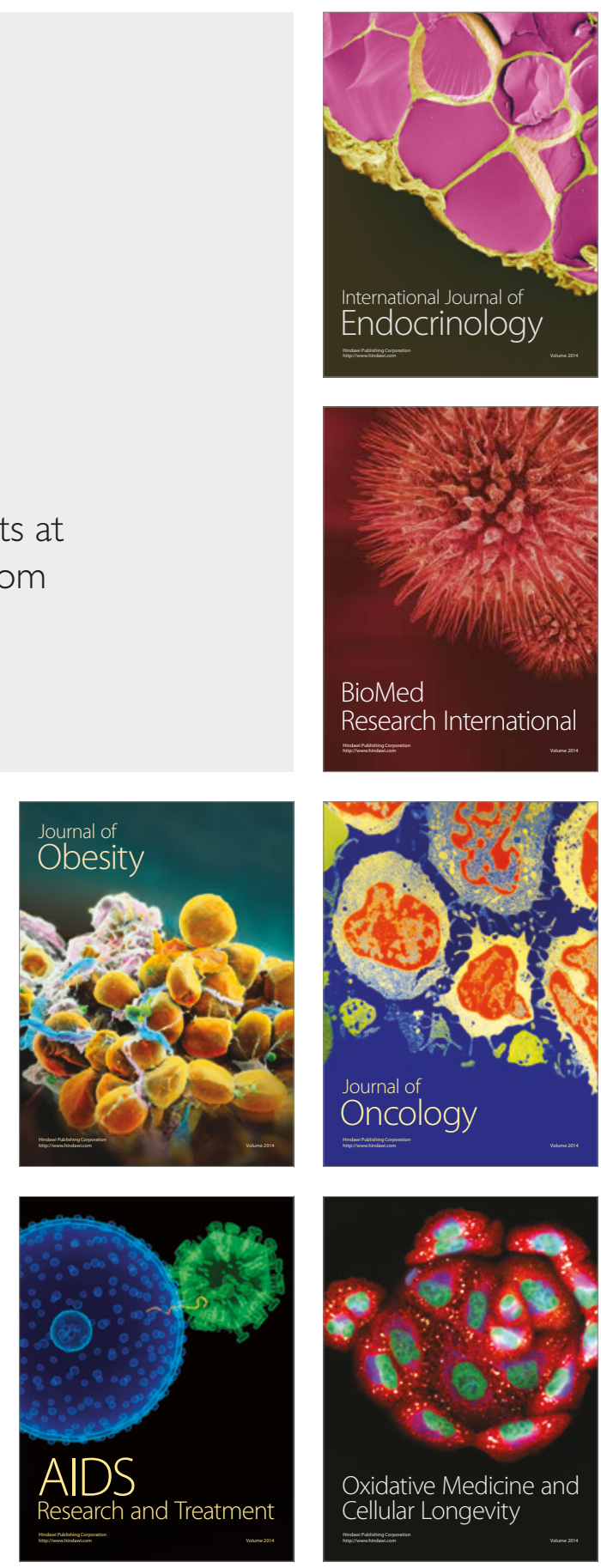